\title{
STATIONARY AND TRANSIENT RESPONSES OF SUSPENSION BRIDGES TO SPATIALLY VARYING GROUND MOTIONS INCLUDING SITE RESPONSE EFFECT
}

\author{
Süleyman Adanur ${ }^{1}$, Ahmet Can Altunışık ${ }^{2, *}$, Kurtuluş Soyluk ${ }^{3}$ and \\ A. Aydın Dumanoğlu ${ }^{4}$ \\ ${ }^{I}$ Assoc. Prof. Karadeniz Technical University, Department of Civil Engineering, Trabzon, Turkey \\ ${ }^{2}$ Karadeniz Technical University, Department of Civil Engineering, Trabzon, Turkey \\ 3 Gazi University, Department of Civil Engineering, Ankara, Turkey \\ 4 Canik Başarı University, Department of Civil Engineering, Samsun, Turkey \\ *(Corresponding author: E-mail: ahmetcan8284@hotmail.com)
}

Received: 21 April 2016; Revised: 3 May 2016; Accepted: 12 May 2016

\begin{abstract}
This paper presents an investigation about the stationary and transient analyses of suspension bridges subjected to spatially varying ground motions including the site response effect. The Bosphorus Suspension Bridge, which connects Europe to Asia in Istanbul, Turkey is selected as a numerical example. The spatial variability of ground motions between the support points is taken into consideration with the coherency function, which arises from three sources: incoherence, wave-passage and site-response effects. The Heaviside Modulating Function has been used throughout the study for computing the transient responses. At the end of the study, the results are compared with each other in two groups as homogeneous-heterogeneous and stationary-transient responses. It is observed that the response values obtained for the heterogeneous soil condition cause larger response values than those of the homogeneous soil condition. Also the greater the differences between the soil conditions, the greater the response values. It is also noticed that the stationary response values are larger than those of the transient responses. Based on the obtained results, the stationary assumption can be accepted as satisfactory for the considered ground motion duration.
\end{abstract}

Keywords: Bosphorus suspension bridge; Incoherence effect; Site-response effect; Spatially varying ground motions; Stationary response; Transient response; Wave-passage effect.

DOI: 10.18057/IJASC.2017.13.4.4

\section{INTRODUCTION}

In long span engineering structures such as suspension bridges, earthquake motions will not be the same over distances due to the complex nature of the earth crust. It is evident that, because of the difference in local soil conditions at the supports, loss of coherency due to reflections and refractions, and travelling with finite velocity between support points, earthquake motions will be subjected to significant variations at the support points of the bridge. This variation will cause internal forces due to the pseudo-static displacements which do not arise under uniform ground motions. So, when analysing suspension bridges, the spatial variability of the earthquake motions should be considered. The spatially varying ground motion can be considered as multiple-support excitation for long span multi-support structural systems.

The earthquake response analysis of long span bridges subjected to spatially varying ground motions has aroused particular interest over the last four decades. The effects of multiple-support seismic excitations on suspension bridges were investigated by Abdel-Ghaffar and Rubin [1,2], who concluded that uncorrelated ground motions overestimate the responses compared to those of the uniform ground motion case. The responses of simplified bridge models such as continuous 
beams, reinforced concrete bridges, and incompressible circular arches to spatially varying ground motions were investigated by Harichandran and Wang [3], Bilici et al. [4], Zhang et al. [5], Zembaty [6], Gao et al. [7], Li and Chouw [8] and the significance of spatially varying ground motions was observed. Simplified bridge models and a suspension bridge model subjected to the spatially varying earthquake motions were studied, by Der Kiureghian and Neuenhofer [9], Nakamura et al. [10], Der Kiureghian et al. [11] based on a newly developed multiple support response spectrum method. They concluded that the developed response spectrum method offers a simple and viable alternative for seismic analysis of multiple supported structures subjected to spatially varying ground motions. The response of a long span suspension bridge to spatially varying ground motion due to topographic effects was analysed by Rassem et al. [12] who concluded that soil conditions, site topography and bridge support locations in a valley are important-factors which demand a more thorough evaluation of the ground motions exciting the bridge. Stochastic response analyses of cable-stayed bridges subjected to spatially varying ground motions were carried out by Allam and Datta [13,14], Soyluk and Sicacık [15], Ateş et al. [16], and Zhang et al. [17] which underlined the significance of the spatial variability of ground motions between the support points.

In random vibration analysis, statistical averages are assumed to be independent of time for stationary excitation. In fact, earthquake motions cannot be stationary, because they initially grow from zero, then have a steady phase and eventually decay. Therefore, earthquake motions should be taken as nonstationary excitations. Although earthquake excitation cannot be stationary throughout the motion, it can be taken as stationary for the time period where maximum structural responses occur.

Stationary and transient response analyses of long span bridges subjected to spatially varying ground motions have been carried out frequently in recent years. Stationary and nonstationary stochastic response analyses of suspension, deck arch and cable-stayed bridges subjected to spatially varying ground motions for homogeneous soil conditions were performed by Hawwari [18], Harichandran et al. [19], Adanur et al. [20], Jia et al. [21], and Soyluk and Dumanoglu [22]. In all of these studies the significance of the spatially varying ground motion was underlined. The nonstationary response of a viaduct subjected to spatially varying ground motions was investigated by Perotti [23] and concluded that the incoherence effect is more important than the wave-passage effect. Hyun et al. [24] developed a new method for the nonstationary response analysis of suspension bridges. Numerical results of this study indicated that correlation effects at different support points have significant effects on the dynamic responses of suspension bridges.

The objective of this paper is to determine the stationary and transient responses of suspension bridges, which have not been analysed comprehensively for spatially varying ground motions including the incoherence, wave-passage and site-response effects together. For this purpose the stationary and transient responses of suspension bridges subjected to spatially varying ground motions are investigated, taking into consideration the site-response, incoherence and wave-passage effects at the same time. The relative contributions of the pseudo-static, dynamic and covariance components to the total response are also presented. 


\section{RANDOM VIBRATION THEORY FOR SPATIALLY VARYING GROUND MOTION}

\subsection{Stationary Response}

One of the effective methods used to carry out random vibration analysis of structural systems is to use the stationary random process theory. This theory has been used widely in the random vibration analysis of structures. The stationary random process theory provides a reasonable basis for the seismic analysis of multiply supported structures where the spatial variability of the ground motion is comprehensively taken into account and hence used consistently by many researchers for the random vibration analysis of structures under the spatially varying ground motions (Der Kiureghian [25]).

In the random vibration theory, the variance of the ith total response component is expressed as [19]

$$
\sigma_{z_{i}}^{2}=\sigma_{z_{i}}^{2 q s}+\sigma_{z_{i}}^{2 d}+2 \operatorname{Cov}\left(z_{i}^{q s}, z_{i}^{d}\right)
$$

where, $\sigma_{z_{i}}^{2 q s}$ is the variance of the pseudo-static response component, $\sigma_{z_{i}}^{2 d}$ is the variance of the dynamic response component and $\operatorname{Cov}\left(z_{i}^{q s}, z_{i}^{d}\right)$ is the covariance between the pseudo-static and dynamic components and can be written as

$$
\begin{aligned}
& \sigma_{z_{i}}^{2 s}=\sum_{l=1}^{r} \sum_{m=1}^{r} A_{i l} A_{i m} \int_{-\infty}^{\infty} \frac{1}{\omega^{4}} S_{\ddot{v}_{g l} \ddot{g}_{g_{m}}}(\omega) d \omega \\
& \sigma_{z_{i}}^{2 d}=\sum_{j=1}^{n} \sum_{k=1}^{n} \sum_{l=1}^{r} \sum_{m=1}^{r} \psi_{i j} \psi_{i k} \Gamma_{l j} \Gamma_{m k} \int_{-\infty}^{\infty} H_{j}(-\omega) H_{k}(\omega) S_{\ddot{v}_{g l} \ddot{v}_{g_{m}}}(\omega) d \omega \\
& \operatorname{Cov}\left(z_{i}^{q s}, z_{i}^{d}\right)=-\sum_{j=1}^{n} \sum_{l=1}^{r} \sum_{m=1}^{r} \psi_{i j} A_{i l} \Gamma_{m j} \int_{-\infty}^{\infty} \frac{1}{\omega^{2}} H_{j}(\omega) S_{\ddot{v}_{g_{l}} \ddot{v}_{g m}}(\omega) d \omega
\end{aligned}
$$

where $\omega$ is the circular frequency, $r$ is the number of support degrees of freedom where the ground motion is applied, $n$ is the number of modes used in the analysis, $A_{i l}$ and $A_{i m}$ are the static displacement components due to unit support motions, $S_{\ddot{v}_{g_{l}} \ddot{v}_{m}}(\omega)$ is the cross spectral density function of accelerations between supports $l$ and $m, \Gamma$ is the modal participation factor, $\psi$ is the eigenvectors and $H(\omega)$ is the frequency response function. The frequency response function is defined as

$$
H_{k}=\frac{1}{\omega_{k}^{2}-\omega^{2}+2 i \xi_{k} \omega_{k} \omega}
$$

where $\omega_{k}$ is the modal circular frequency and $\xi_{k}$ is the modal damping ratio.

\subsection{Transient Response}

It is well known that it is rather complicated and tedious to get closed-from expressions for the modal frequency response function to model the non-stationarity of the ground accelerations. Because the main objective of this study is to determine the stationary and transient responses of suspension bridges qualitatively, the Heaviside Modulating Function is used for the modal 
frequency response function to determine the transient structural responses (Harichandran et al. [19]).

For the present study, the Heaviside Modulating Function is used to take into account the effect of transient responses. The Heaviside Modulating Function is defined as [19]

$H_{k}(\omega, t)=H_{k}(\omega)\left\{1-\exp \left(-\xi_{k} \omega_{k} t\right) \exp (-i \omega t)\left[\cos \omega_{k d} t+\frac{\left(\xi_{k} \omega_{k}+i \omega\right)}{\omega_{k d}} \sin \omega_{k d} t\right]\right\}$

where $\omega_{k d}=\omega_{k} \sqrt{1-\xi_{k}^{2}}$. The transient responses at given times are computed by replacing the normal frequency response function with the function $H_{k}(\omega, t)$ in the expressions written for the stationary response.

\subsection{Mean of Maximum Value}

Depending on the peak response and standard deviation of the total response, the mean of maximum value $(\mu)$, in the stochastic analysis can be written as

$\mu=p \sigma_{z}$

where $p$ is a peak factor and $\sigma_{z}$ is the standard deviation of the total response [9].

\section{SPATIALLY VARYING GROUND MOTION MODEL}

The spatial variability of ground motion is characterised by the coherency function in the frequency domain. This function is dimensionless and complex valued. For the coherency function, the following model proposed by Der Kiureghian [25] is used

$\gamma_{l m}(\omega)=\gamma_{l m}(\omega)^{i} \gamma_{l m}(\omega)^{w} \gamma_{l m}(\omega)^{s}=\gamma_{l m}(\omega)^{i} \exp \left[i\left(\theta_{l m}(\omega)^{w}+\theta_{l m}(\omega)^{s}\right)\right]$

where $\gamma_{l m}(\omega)^{i}$ characterises the real valued incoherence effect, $\gamma_{l m}(\omega)^{w}$ indicates the complex valued wave-passage effect and $\gamma_{l m}(\omega)^{s}$ defines the complex valued site-response effect.

For the incoherence effect, resulting from reflections and refractions of seismic waves through the soil during their propagation, the widely used model proposed by Harichandran and Vanmarcke [26] is considered. This model is based on the analysis of recordings made by the SMART-1 seismograph array in Lotung, Taiwan and defined as

$$
\begin{aligned}
& \gamma_{l m}(\omega)^{i}=A \exp \left[-\frac{2 d_{l m}}{\alpha \theta(\omega)}(1-A+\alpha A)\right]+(1-A) \exp \left[-\frac{2 d_{l m}}{\theta(\omega)}(1-A+\alpha A)\right] \\
& \theta(\omega)=k\left[1+\left(\frac{\omega}{2 \pi f_{0}}\right)^{b}\right]^{-\frac{1}{2}}
\end{aligned}
$$

where $d_{l m}$ is the distance between support points $l$ and $m . A, \alpha, k, f_{0}$ and $b$ are model parameters and 
in this study the values obtained by Harichandran et al. [19] are used $(A=0.636, \alpha=0.0186, k=31200$, $f_{0}=1.51 \mathrm{~Hz}$ and $\left.b=2.95\right)$.

The wave-passage effect resulting from the difference in the arrival times of waves at support points is defined as [25]

$\theta_{l m}(\omega)^{w}=-\frac{\omega d_{l m}^{L}}{v_{a p p}}$

where $v_{\text {app }}$ is the apparent wave velocity and $d_{l m}^{L}$ is the projection of $d_{l m}$ on the ground surface along the direction of propagation of the seismic waves. The apparent wave velocities employed in this study are $v_{\text {app }}=400 \mathrm{~m} / \mathrm{s}$ for soft soil, $v_{\text {app }}=700 \mathrm{~m} / \mathrm{s}$ for medium soil and $v_{\text {app }}=1000 \mathrm{~m} / \mathrm{s}$ for firm soil.

The site-response effect resulting from the differences in local soil conditions at the support points is defined as [25]

$\theta_{l m}(\omega)^{s}=\tan ^{-1} \frac{\operatorname{Im}\left[H_{l}(\omega) H_{m}(-\omega)\right]}{\operatorname{Re}\left[H_{l}(\omega) H_{m}(-\omega)\right]}$

where $H_{l}(w)$ is the local soil frequency response function representing the filtration through soil layers.

The power spectral density function of the ground acceleration $\left(\ddot{v}_{g_{l}}\right)$ characterising the earthquake process is assumed to be of the following form modified by Clough and Penzien [27].

$S_{\ddot{v}_{g l} \ddot{g}_{g l}}(\omega)=S_{0}\left[\frac{\omega_{l}^{4}+4 \xi_{l}^{2} \omega_{l}^{2} \omega^{2}}{\left(\omega_{l}^{2}-\omega^{2}\right)^{2}+4 \xi_{l}^{2} \omega_{l}^{2} \omega^{2}}\right]\left[\frac{\omega^{4}}{\left(\omega_{f}^{2}-\omega^{2}\right)^{2}+4 \xi_{f}^{2} \omega_{f}^{2} \omega^{2}}\right]$

where $S_{0}$ is the amplitude of the white-noise bedrock acceleration, and $\omega_{l}, \xi_{l}$ and $\omega_{f}, \xi_{f}$ are the resonant frequency and damping ratio of the first and second filters, respectively.

In this study, firm (F), medium (M) and soft (S) soil types are used. The filter parameters for these soil types proposed by Der Kiureghian and Neuenhofer [9] are utilized as presented in Table 1. The amplitude of the white-noise bedrock acceleration (S0) is obtained for each soil type by equating the variance of the ground acceleration to the variance of the S16E component of the Pacoima Dam acceleration records of the 1971 San Fernando earthquake. The calculated values of the intensity parameter for each soil type are $\mathrm{S} 0$ (firm) $=0.009715 \mathrm{~m} 2 / \mathrm{s} 3, \mathrm{~S} 0$ (medium) $=0.014436 \mathrm{~m} 2 / \mathrm{s} 3$, and $\mathrm{S} 0$ (soft) $=0.020267 \mathrm{~m} 2 / \mathrm{s} 3$. Acceleration power spectral density function for each soil type is presented in Figure 1.

Table 1. Power Spectral Density Parameters for Model Soil Types

\begin{tabular}{lcccc}
\hline Soil Type & $\omega_{l}(\mathrm{rad} / \mathrm{s})$ & $\xi_{g}$ & $\omega_{f}(\mathrm{rad} / \mathrm{s})$ & $\xi_{f}$ \\
\hline Firm & 15.0 & 0.6 & 1.5 & 0.6 \\
Medium & 10.0 & 0.4 & 1.0 & 0.6 \\
Soft & 5.0 & 0.2 & 0.5 & 0.6 \\
\hline
\end{tabular}




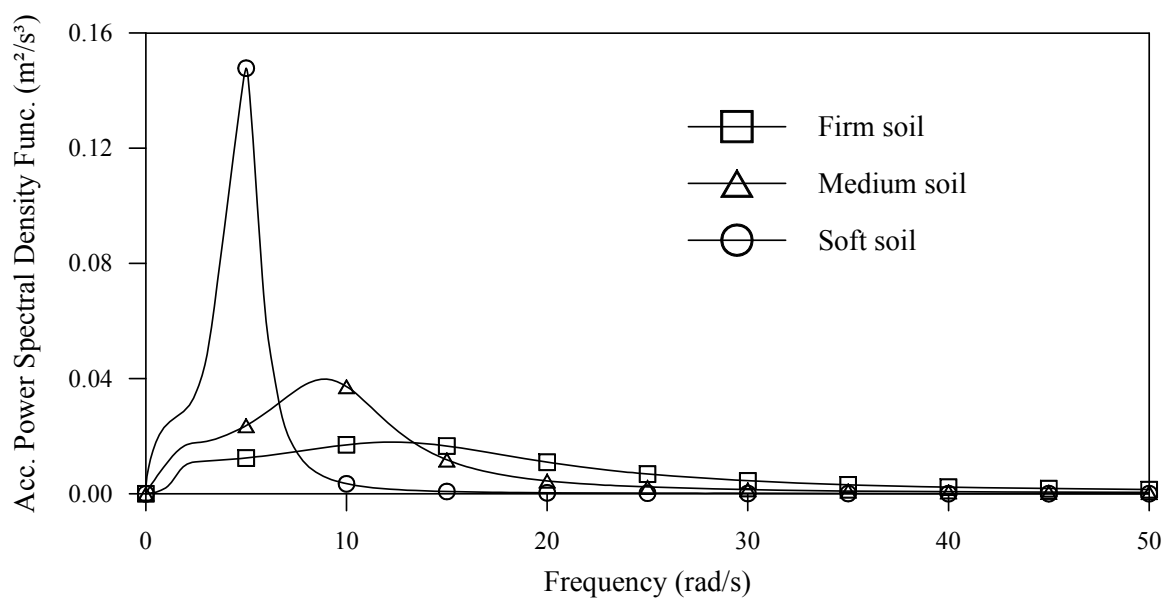

Figure 1. Acceleration Power Spectral Density Functions for the Filtered White Noise Model for Different Soil

\section{NUMERICAL EXAMPLE}

In this study, the Bosphorus Suspension Bridge in Turkey which connects Europe to Asia in Istanbul is selected as a numerical example (Figure 2). The construction of the bridge started in 1970 and was completed in 1973.

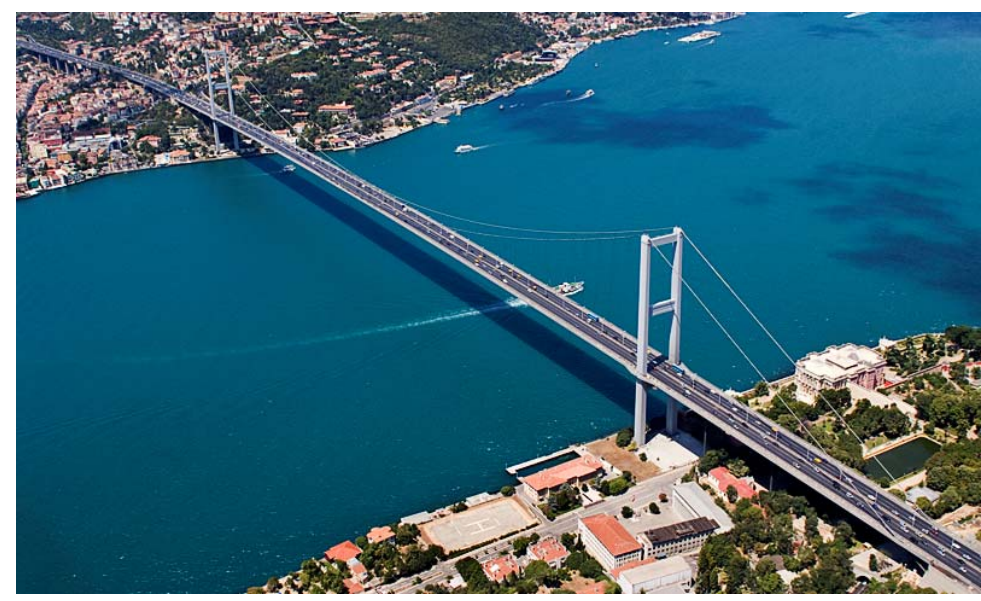

Figure 2. Bosphorus Suspension Bridge

The bridge has steel towers which are flexible, inclined hangers and a steel box-deck of $1074 \mathrm{~m}$ main span with side spans of 231 and $255 \mathrm{~m}$ on the European and Asian sides, respectively. The horizontal distance between the cables is $28 \mathrm{~m}$ and the roadway is $21 \mathrm{~m}$ wide, accommodating three lanes each way. The roadway at the mid-span of the bridge is approximately $64 \mathrm{~m}$ above the sea level. A schematic representation of the Bosphorus Suspension Bridge including the dimensions is given in Figure 3. 


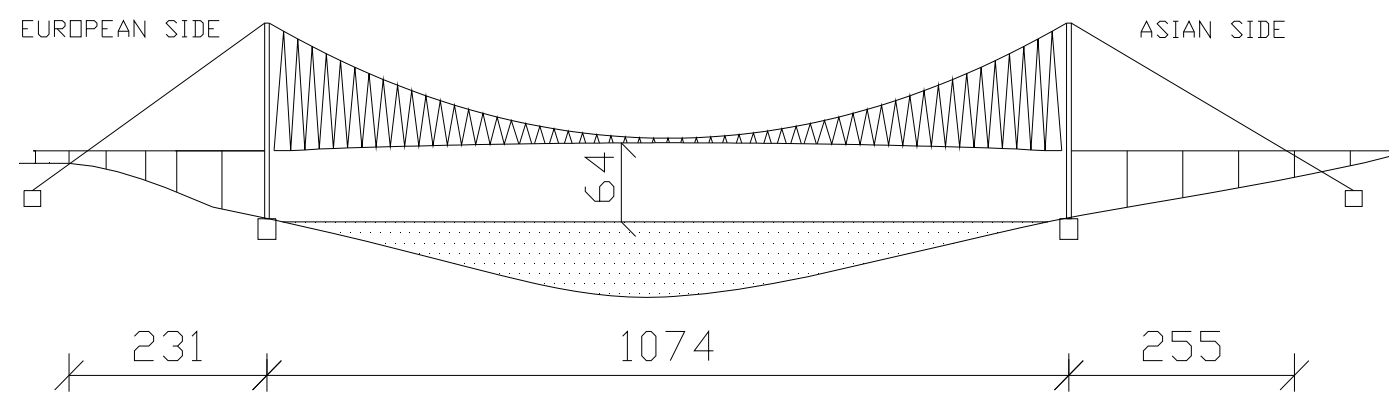

Figure 3. Schematic Representation of the Bosphorus Bridge Including the Dimensions

(Dimensions as m)

The deck was constituted with particular concern for aerodynamic form in order to reduce the wind effect along the bridge deck. The aerodynamic steel box-girder deck of the bridge consists of 60 box-girder deck pieces, each comprising $3 \mathrm{~m}$ deep prefabricated sections of $17.9 \mathrm{~m}$ long $\mathrm{x} 33 \mathrm{~m}$. The top of each box section consists of an orthotropic plate on which a $35 \mathrm{~mm}$ thick mastic asphalt surface is laid.

The bridge has $165 \mathrm{~m}$ high slender steel towers. The tower legs are $5.20 \times 7.00 \mathrm{~m}$ at the bottom and they taper to $3.00 \times 7.00 \mathrm{~m}$ at the top. Vertical tower legs are connected by three horizontal portal beams. Dimensions of the bridge towers are given in Figure 4.
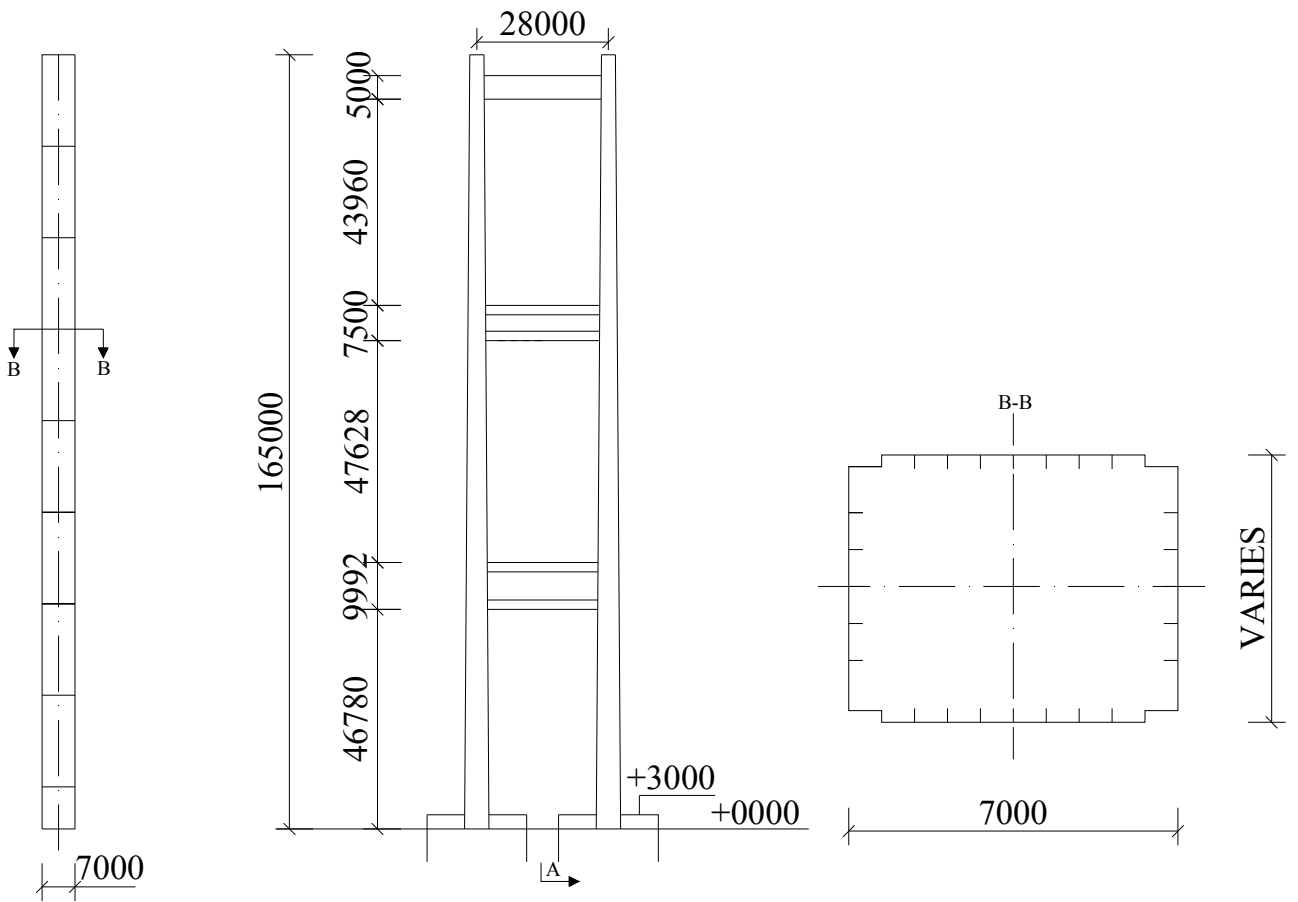

Figure 4. Dimensions of the Bridge Towers (Dimensions as $\mathrm{mm}$ )

The main cables of the bridge are built up from parallel wire, $5 \mathrm{~mm}$ in diameter over the hot dipped galvanizing. Each main cable consists of 19 strands extending between the towers and contains 548 parallel wires, with a further four strands, each containing 192 wires for the backstays.

\subsection{Mathematical Model of the Bridge}

To investigate the stochastic response of the Bosphorus Suspension Bridge, a two-dimensional 
mathematical model of the bridge with 202 nodes, 159 beam elements, 118 truss elements, and 475 degrees of freedom is used in the analyses (Figure 5). The deck, towers, and cables of the selected bridge are modelled using beam elements and the hangers are modelled by truss elements. The analyses are obtained for a $2.5 \%$ damping ratio and for the first 15 modes. The stiffening effects of the cables caused by dead load are also accounted for in the analyses.
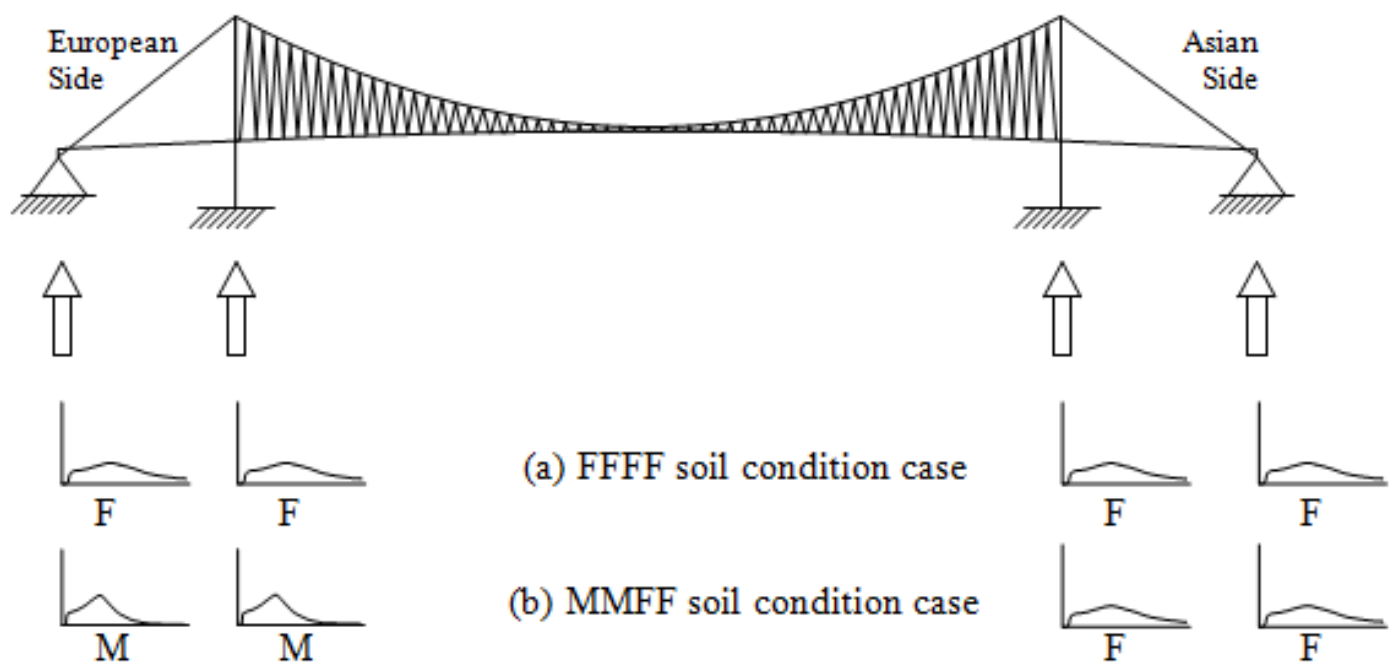

(b) MMFF soil condition case
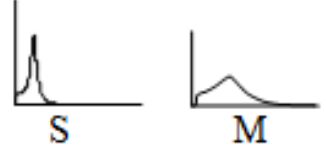

(c) SMFF soil condition case

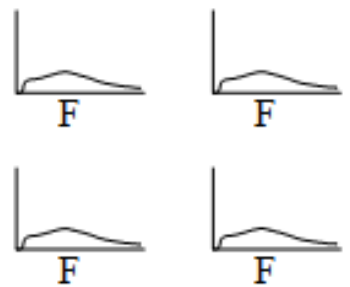

Figure 5. Suspension Bridge Subjected to Spatially Varying Ground Motions in the Vertical Direction for Homogeneous and Heterogeneous Soil Conditions.

\subsection{Numerical computations}

Stationary and transient analyses of a suspension bridge subjected to spatially varying ground motions are carried out taking into account the incoherence, wave-passage and site-response effects. For this purpose the following three different soil condition cases are considered. Each letter corresponds to a particular support and the soil conditions at that support point.

Case A: All the supports are assumed to be founded on soils with firm soil type (FFFF). This case corresponds to the homogeneous soil type.

Case B: While the supports at the European side are assumed to be founded on medium soil, the supports at the Asian side are assumed to be founded on firm soil type (MMFF).

Case C: The European side anchorage is founded on soft soil, the European side tower pier is founded on medium soil and the supports of the bridge at the Asian side are founded on firm soil (SMFF).

The filtered white noise ground motion model modified by Clough and Penzien [27] is used. It is applied in the vertical direction as a ground motion model in which the spectral density function intensity parameter is determined according to the S16E component of the Pacoima dam record of the San Fernando earthquake in 1971.

The suspension bridge subjected to spatially varying ground motion in the vertical direction for homogeneous and heterogeneous soil cases is shown in Figure 5. The vertical input is assumed to travel across the bridge from the European side to the Asian side with finite velocity of $400 \mathrm{~m} / \mathrm{s}$ for soft soil, $700 \mathrm{~m} / \mathrm{s}$ for medium soil and $1000 \mathrm{~m} / \mathrm{s}$ for firm soil. The spectral density function applied 
to each support point as a ground motion is different for each soil type. The general excitation case, which includes the three spatial variability effects, namely the incoherence, wave-passage and site-response effects, is considered in this study.

To determine whether the bridge will reach its stationary response during various durations of the strong shaking; the variances of the transient responses are calculated at 10, 20, 30 and 40 seconds and compared with the stationary responses for the spatially varying ground motions.

\section{NUMERICAL RESULTS}

\subsection{Comparison of Homogeneous and Heterogeneous Responses}

The mean of maximum values of total responses is carried out for the considered soil condition sets under the general excitation case which includes the most important effects of spatially varying ground motions. The mean of maximum total vertical deck displacements, bending moments and shear forces calculated for different soil condition sets, defined as FFFF, MMFF and SMFF, are compared in Figure 6. As can be observed from these figures, the stationary response values obtained for the FFFF (homogeneous) soil condition set are the lowest, and the response values for SMFF soil condition set are the highest. The total displacements and bending moments at the middle of the deck obtained from the general excitation case overestimates the responses by $68.39 \%, 98.41 \%$ and by $39.64 \%, 51.46 \%$ for the MMFF, and SMFF soil condition cases, respectively, when compared with the responses due to the FFFF (homogeneous) soil condition case. At the deck point where maximum shear forces take place it is found that the total shear forces obtained from the general excitation case overestimate the response by $52.94 \%$, and $67.48 \%$ for the MMFF, and SMFF soil condition cases, respectively, when compared with the response due to the FFFF soil condition case. While the total vertical displacements obtained at the end of deck on the Asian side are close to each other for the three different soil condition sets, the displacements obtained at the end of deck on the European side for the MMFF and SMFF soil condition sets are larger than those of the FFFF soil condition case. This is due to the change in the soil conditions at the European side from firm to soft.

The mean of the maximum total horizontal displacements, bending moments and shear forces at the European and Asian side towers obtained for the three different soil condition sets under the general excitation case are presented in Figure 7. It is evident that the response values obtained at the tower on the Asian side are very much smaller than the response values calculated at the tower on the European side where the soil conditions change from firm to soft. It is also indicated that the response values obtained for the SMFF soil condition case are generally the highest. Moreover, the greater the difference between the soil conditions, generally the greater the response values found at both towers. 


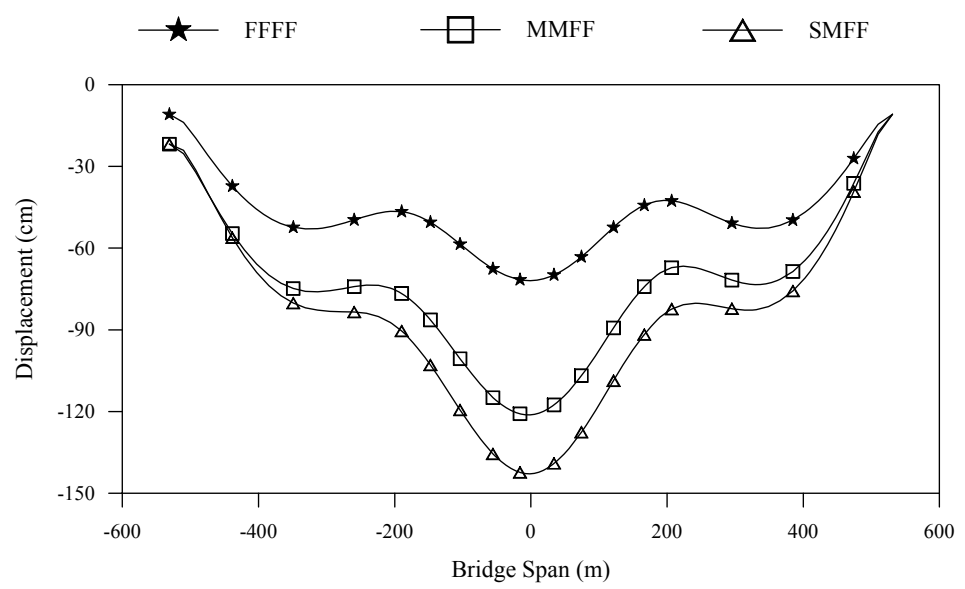

(a) Vertical Displacements

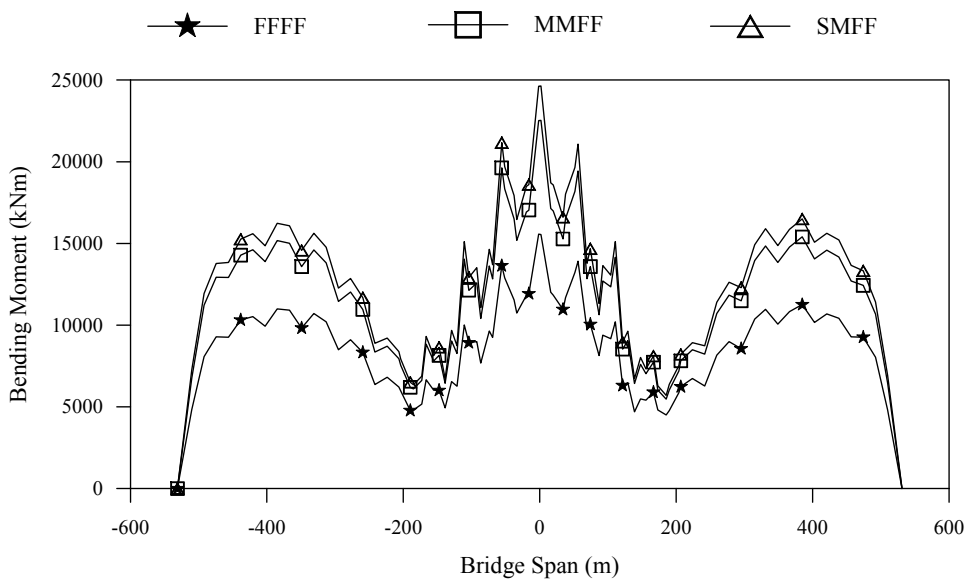

(b) Bending Moments

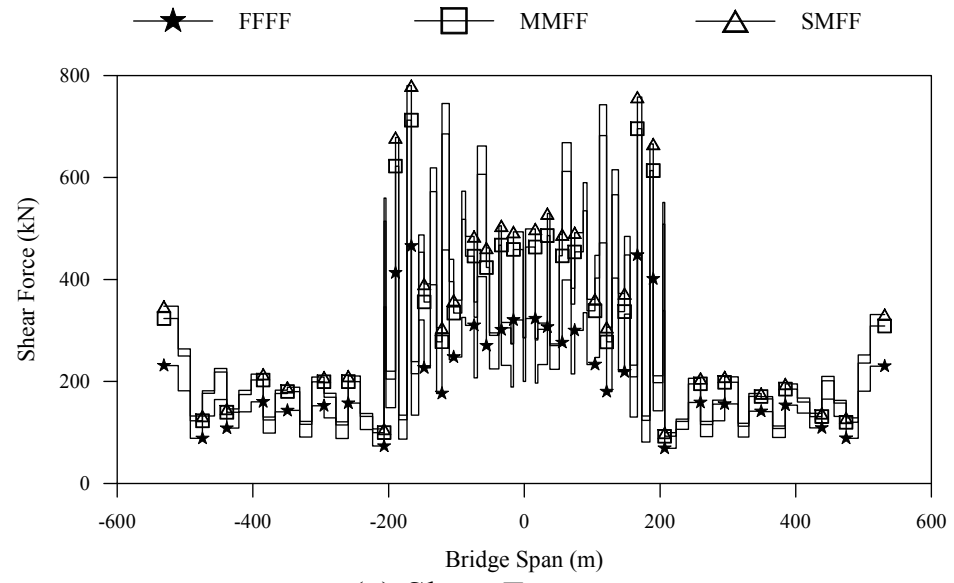

(c) Shear Forces

Figure 6. Mean of Maximum Total Response Values at the Deck for Different Soil Condition Sets under the General Excitation Case 
European Side Tower

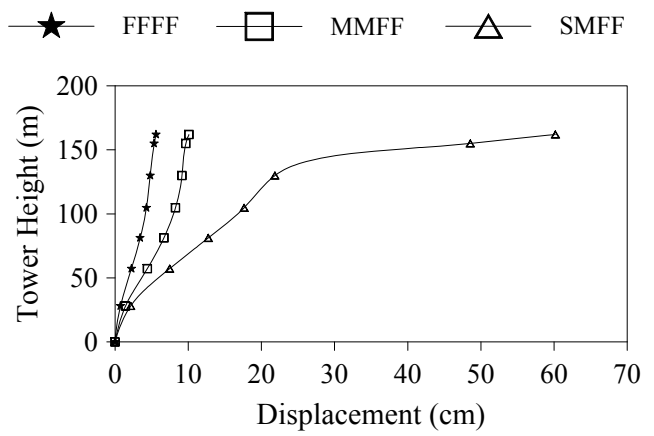

$\underline{\text { Asian Side Tower }}$

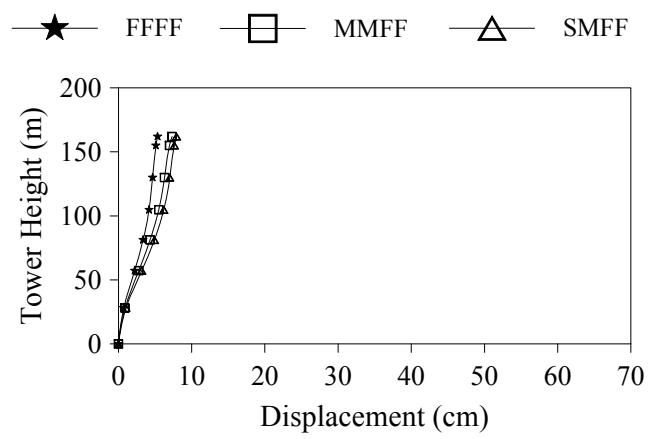

(a) Horizontal Displacements
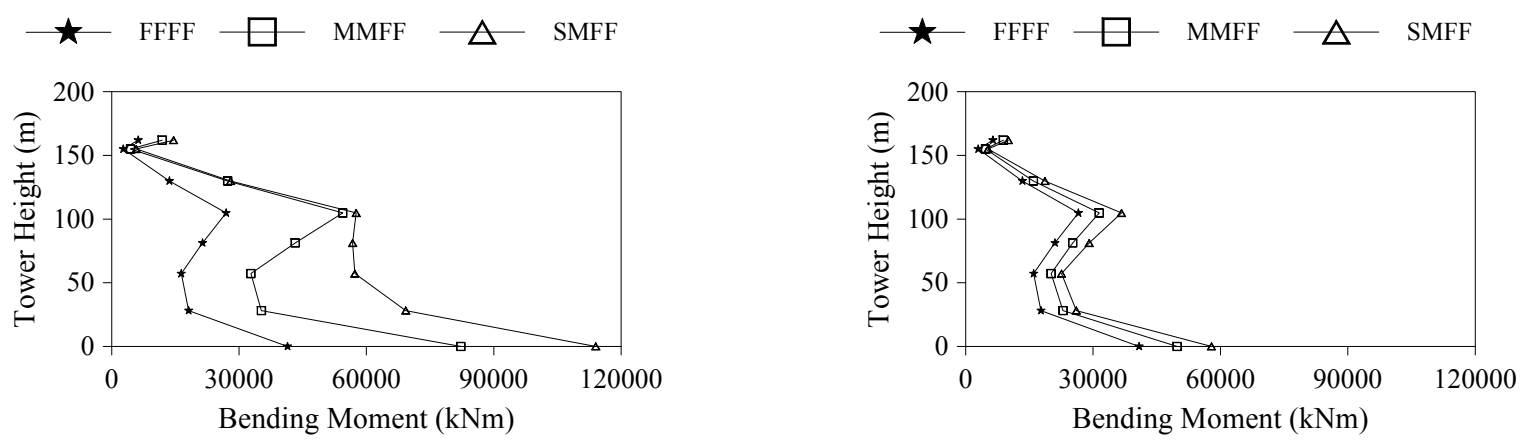

(b) Bending Moments
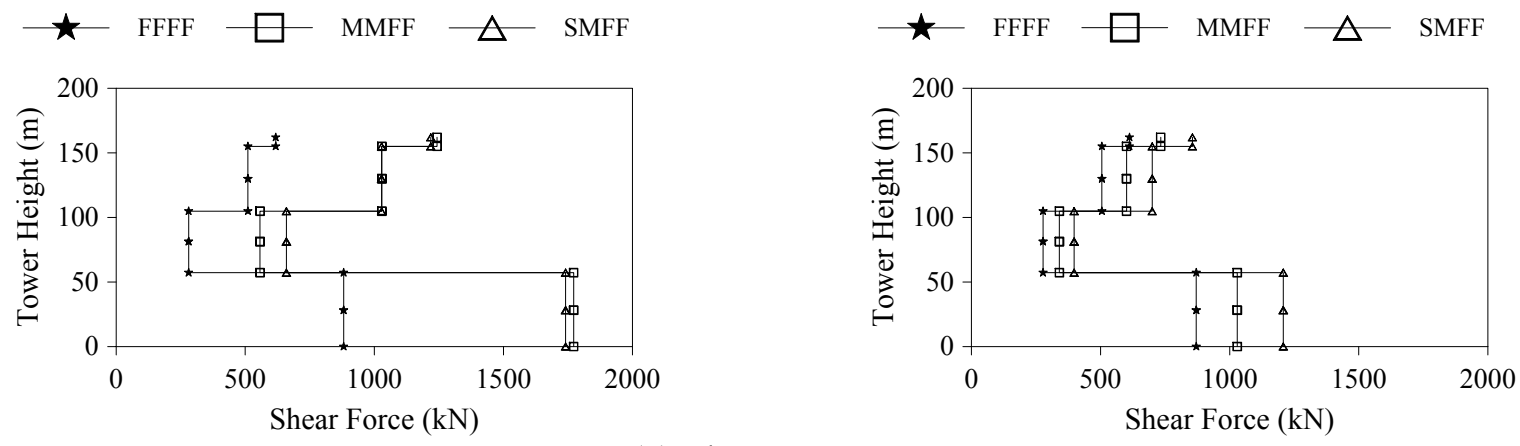

(c) Shear Forces

Figure 7. Mean of Maximum Total Response Values at the European and Asian Side Towers for Different Soil Condition Sets under the General Excitation Case

The variance of total response has three components; the pseudo-static component, the dynamic component and the covariance component between the pseudo-static and dynamic components. In this section, the contribution of each component to the total responses of the bridge is investigated. The process of normalisation is performed by dividing the variance values by the maximum total response. The relative contribution of each component to the total vertical displacement along the bridge deck under the general ground motion case is presented in Figure 8 for the FFFF, MMFF and SMFF soil condition sets. It can be observed that the total displacements are dominated by the dynamic component for all the soil condition sets. However, when the soil condition weakens from firm to soft it can be noticed that the contribution of the dynamic component to the total response decreases, and the contribution of the pseudo-static and covariance components increase. 
The relative contributions of the response components to the total horizontal tower displacements at the European and Asian side towers are shown in Figure 9 under the general excitation case for the three soil condition sets. While the variations obtained for the displacements at the Asian side tower for each soil condition set are similar to each other, the responses obtained at the European side tower show a different variation. At the top point of the European side tower where the maximum total-horizontal displacement takes place, it can be observed that the dynamic component contributes $65.35 \%, 41.00 \%$, and $4.66 \%$; the pseudo-static component contributes $40.79 \%, 55.19 \%$, and $91.86 \%$ and the covariance component contributes $-6.14 \%, 3.81 \%, 3.48 \%$ for the FFFF, MMFF, and SMFF soil condition sets, respectively. Similarly, at the top point of the Asian side tower the dynamic component contributes $76.01 \%, 87.50 \%$, and $92.00 \%$; the pseudo-static component contribute $40.51 \%, 20.23 \%, 18.60 \%$ and the covariance component contributes $-16.52 \%,-7.73 \%$, and $10.60 \%$ for the FFFF, MMFF, and SMFF soil condition sets, respectively. At the European side tower, the total displacements are dominated by the dynamic component for the homogeneous FFFF soil condition case, whereas the total displacements are dominated by the pseudo-static component for the MMFF and SMFF soil condition sets. This can be attributed to the fact that the European side supports are located on weaker soil conditions and hence the pseudo-static components are amplified. The total response is dominated by the dynamic component at the Asian side tower for each soil condition case.

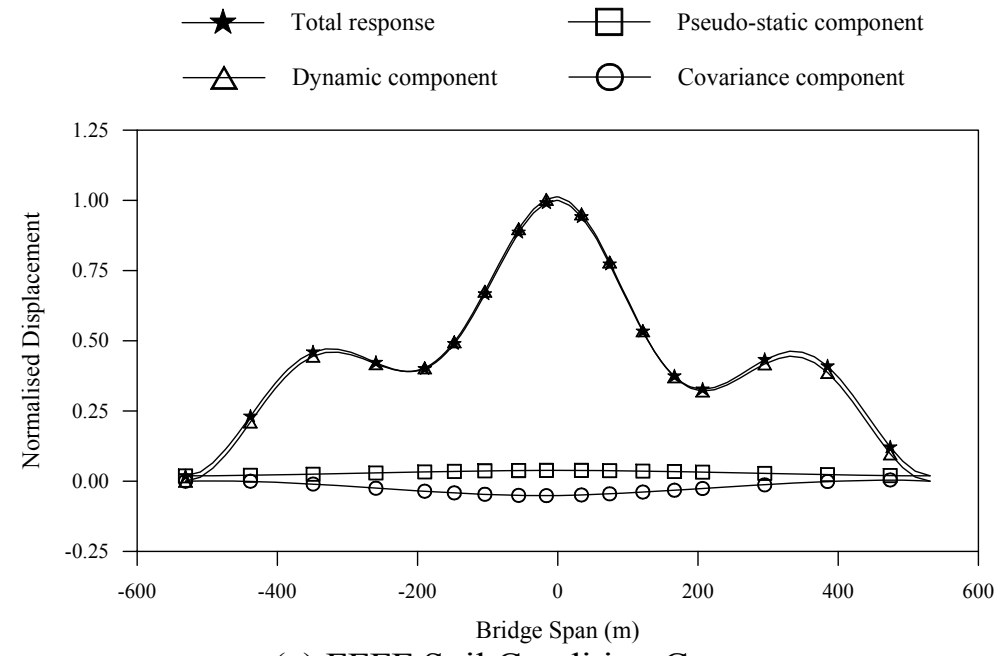

(a) FFFF Soil Condition Case

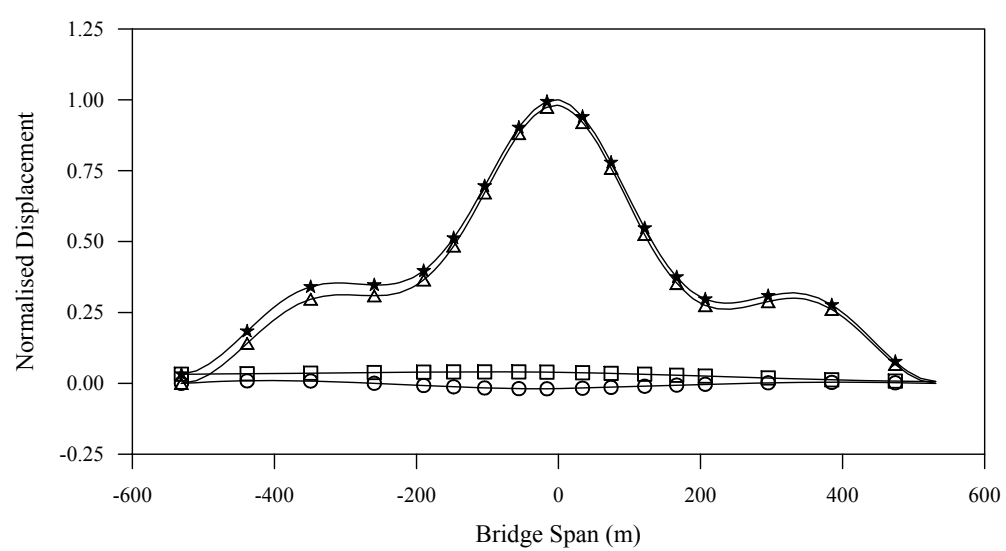

(b) MMFF Soil Condition Case 


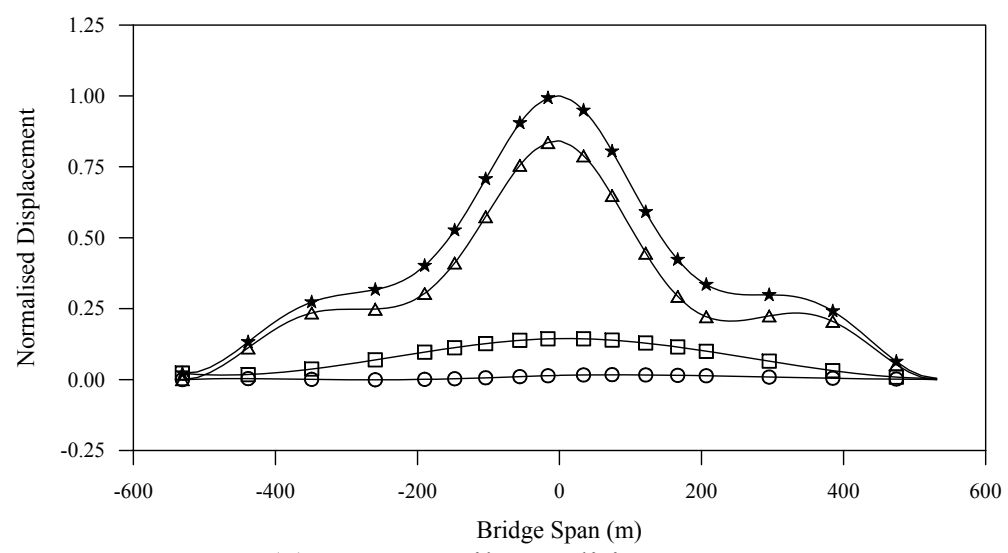

(c) SMFF Soil Condition Case

Figure 8. Normalised Displacement Variances of the Deck for the General Excitation Case

\section{European Side Tower}

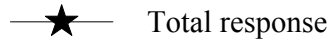

$\square$ Pseudo-static component

$\triangle$ Dynamic component

$\bigcirc$ Covariance component

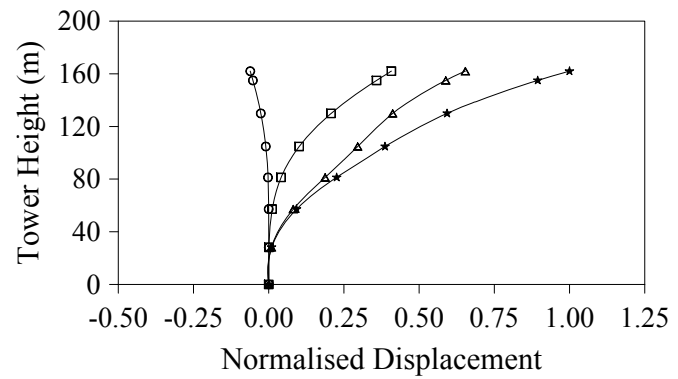

$\underline{\text { Asian Side Tower }}$

$\star \quad$ Total response

$\square$ Pseudo-static component

$\triangle$ Dynamic component

$\bigcirc$ Covariance component

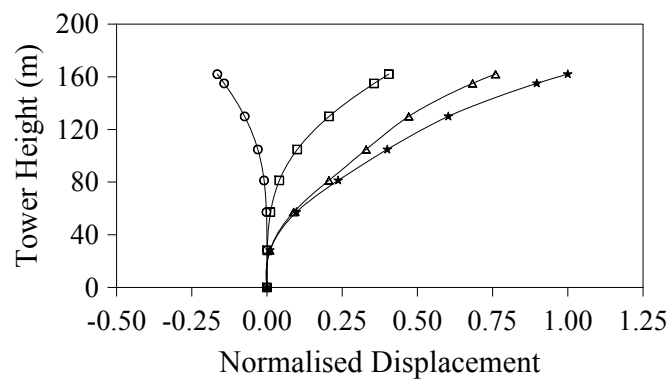

(a) FFFF Soil Condition Case
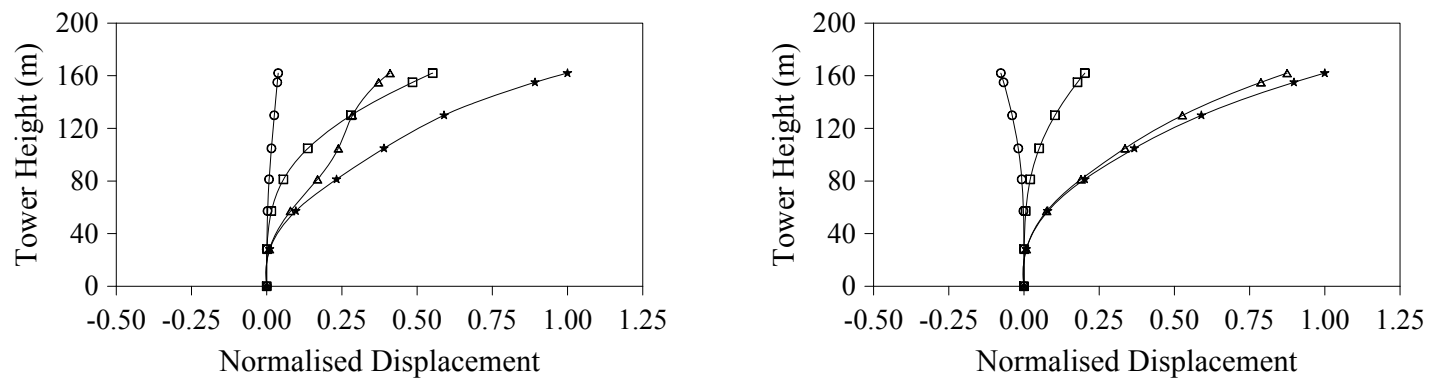

(b) MMFF Soil Condition Case 

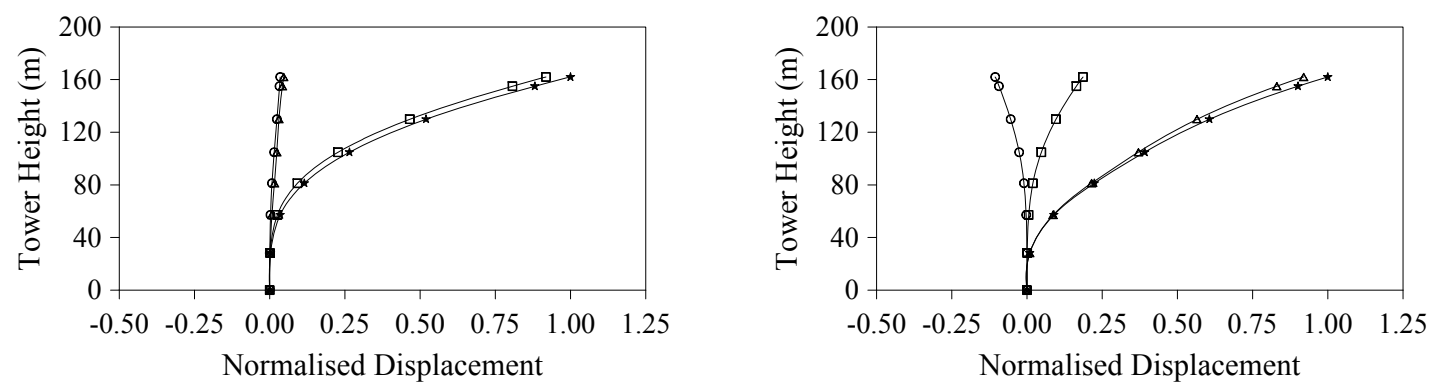

(c) SMFF Soil Condition Case

Figure 9. Normalised Displacement Variances of the European and Asian Side Towers for General Excitation Case

\section{$5.2 \quad$ Comparison of Stationary and Transient Responses}

The normalised response values of the vertical deck displacements, bending moments and shear forces are detailed in Figure 10. The transient responses are calculated along the length of the deck at the designated times of 10,20,30 and $40 \mathrm{sec}$ and compared with the stationary responses for the SMFF soil condition case (general excitation). Each response has been normalised by dividing it by the corresponding maximum stationary response. As can be observed the stationary response values are larger than those of the transient values. For the total displacements obtained at the middle of the deck; $57.44 \%, 73.72 \%, 83.40 \%$ and $89.80 \%$ of the stationary response is achieved at the times of 10, 20, 30 and 40 seconds of the transient response, respectively. For the total bending moments obtained at the middle of the deck where maximum moments take place; $60.84 \%, 70.83 \%, 85.85 \%$ and $90.48 \%$ of the stationary response is achieved at the times of 10, 20, 30 and 40 seconds of the transient response, respectively. For the total shear forces obtained at the deck where maximum shears occur; $52.70 \%, 74.57 \%, 82.56 \%$ and $89.74 \%$ of the stationary response is achieved at the times of 10, 20, 30 and 40 seconds of the transient response, respectively.

Figure 11 shows the normalised response values of the European and Asian side towers for horizontal displacements, bending moments and shear forces at the times of 10, 20, 30 and $40 \mathrm{sec}$ for the transient response as well as for the stationary response under the general excitation case (SMFF soil condition set). It can be observed that while the response values obtained from the transient analysis are close to those of the stationary analysis at the European side tower at the time of $10 \mathrm{sec}$, the response values obtained from the transient analysis are close to those of the stationary analysis at the time of $40 \mathrm{sec}$ for the Asian side tower. The reason can be attributed to the fact that while the pseudo-static component dominates the total responses at the European side tower due to the weaker soil condition case (SM), the dynamic component dominates the total responses at the Asian side tower due to the homogeneous stiffer soil conditions (FF). From this point of view the stationary assumption can be accepted as satisfactory for the considered ground motion duration. 


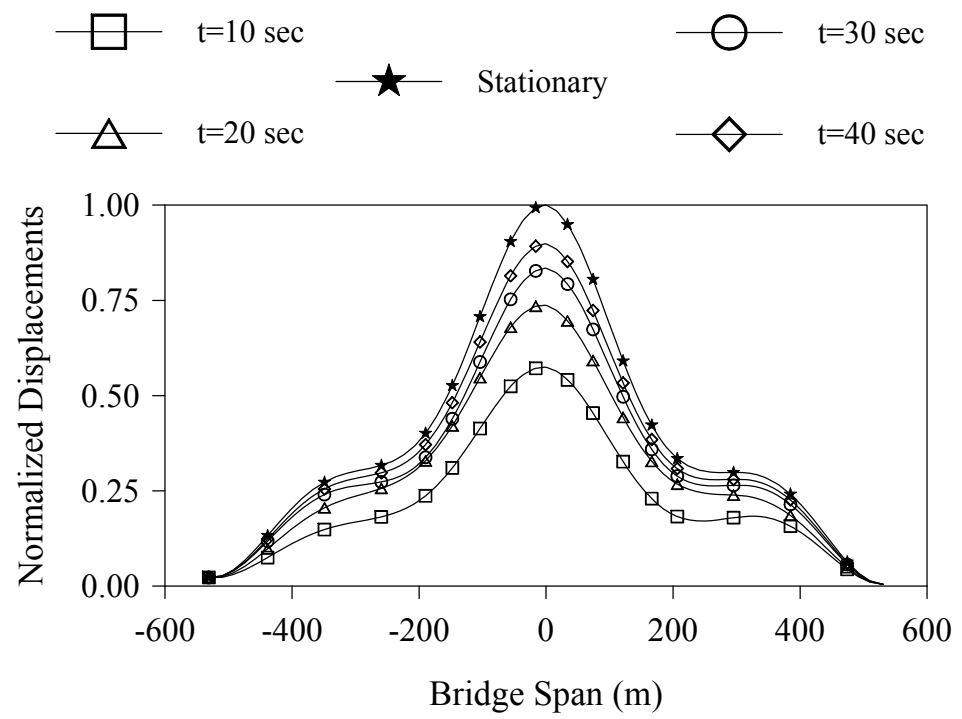

(a) Vertical Displacements

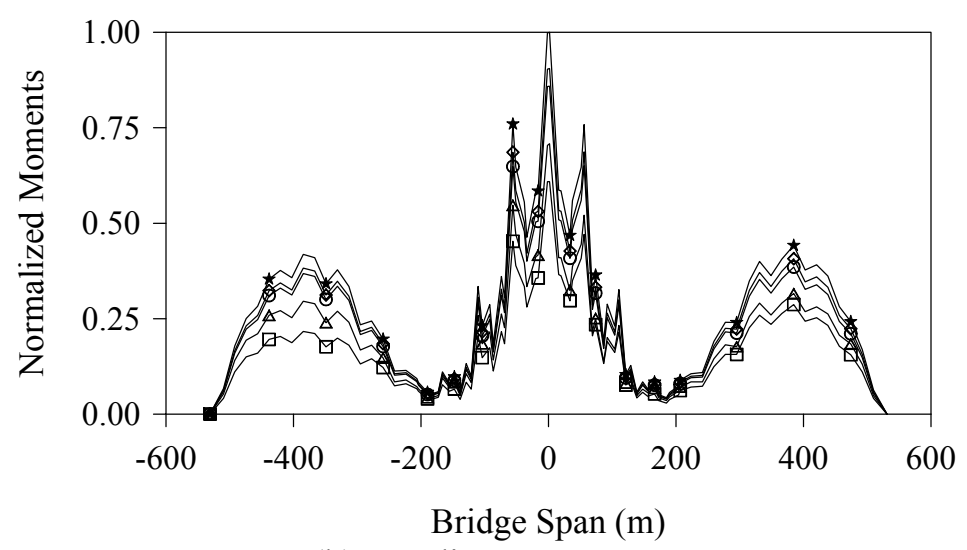

(b) Bending Moments

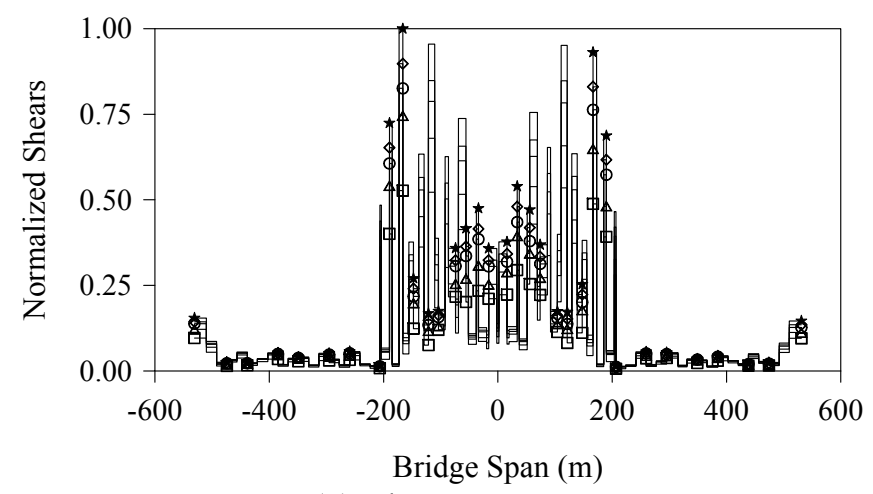

(c) Shear Forces

Figure 10. Normalised Response Values at the Deck Obtained from the Stationary and Transient Analyses under the General Excitation for the SMFF Soil Condition Case 
European Side Tower
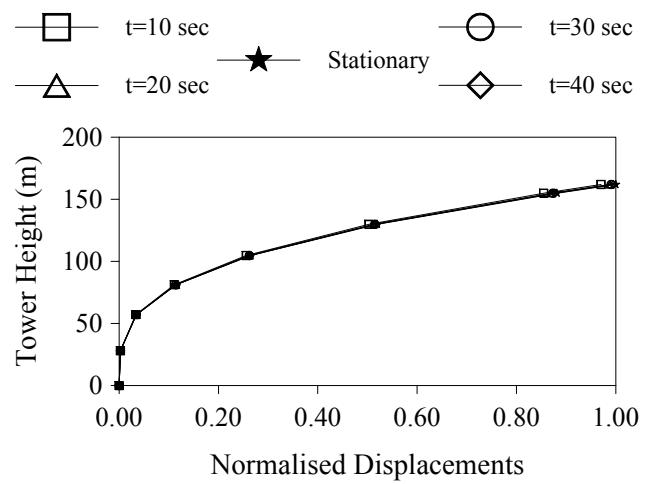

Asian Side Tower
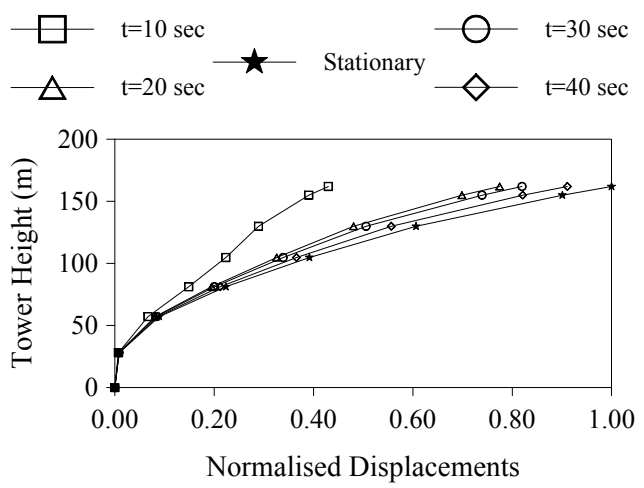

(a) Horizontal displacements
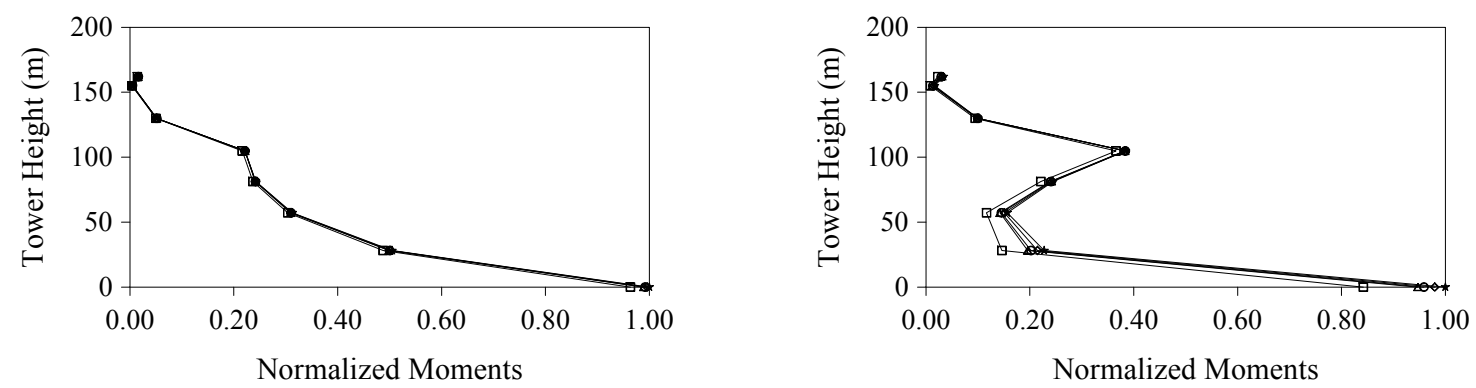

(b) Bending moments
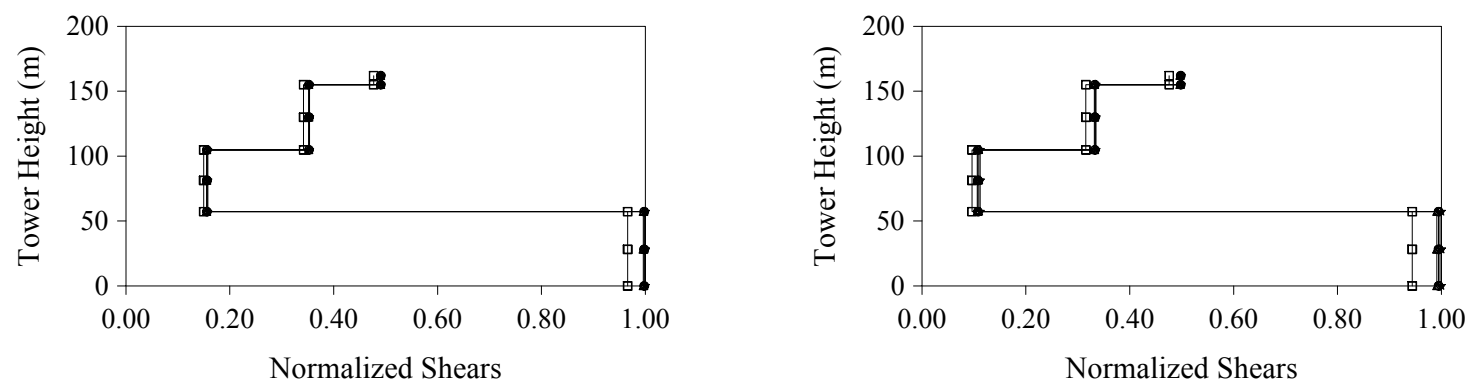

(c) Shear forces

Figure 11. Normalised Response Values at the European and Asian Side Towers Obtained from Stationary and Transient Analyses under the General Excitation for the SMFF Soil

Condition Case

The relative contributions of the pseudo-static, dynamic and covariance components to the total displacement responses at the deck, at the European side tower and at the Asian side tower for the stationary analysis and transient analysis at $\mathrm{t}=10 \mathrm{sec}$ are presented in Figures 12 and 13, respectively. It can be observed that the variation of the transient response is generally consistent with the variation of the stationary response. Because the stationary displacements are generally larger than those of the transient displacements and the pseudo-static displacements are equal for both responses, the contribution of the transient pseudo-static displacements to the total response is increased and the contribution of the transient dynamic components is decreased when compared with those of the stationary contributions. This situation can be observed very clearly on the deck and at the Asian side tower and less noticeably at the European side tower where the total response values are dominated by the pseudo-static component depending on the weaker soil conditions. At the middle of the deck, the contributions of the pseudo-static, dynamic and covariance components to the total response are $14.44 \%, 84.12 \%$ and $1.44 \%$ for the stationary analysis and $25.14 \%$, 
$72.70 \%$ and $2.16 \%$ for the transient analysis, respectively. At the top of the Asian side tower, the contributions of the pseudo-static, dynamic and covariance components to the total response are $18.60 \%, 92.00 \%$ and $-10.60 \%$ for the stationary analysis and $43.28 \%, 81.37 \%$ and $-24.65 \%$ for the transient analysis, respectively. The ratios obtained at the top point of the European side tower are $91.86 \%, 4.66 \%$ and $3.48 \%$ for the stationary analysis and $94.66 \%, 1.81 \%$ and $3.53 \%$ for the transient analysis, respectively.
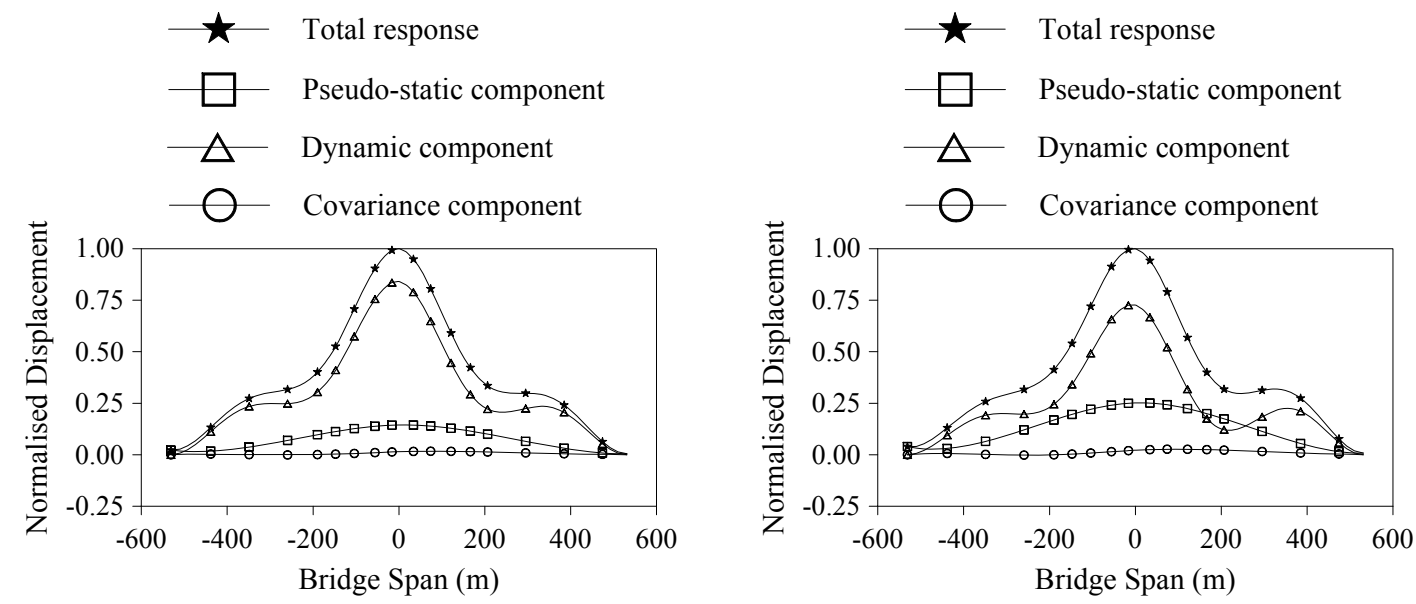

(a) Deck
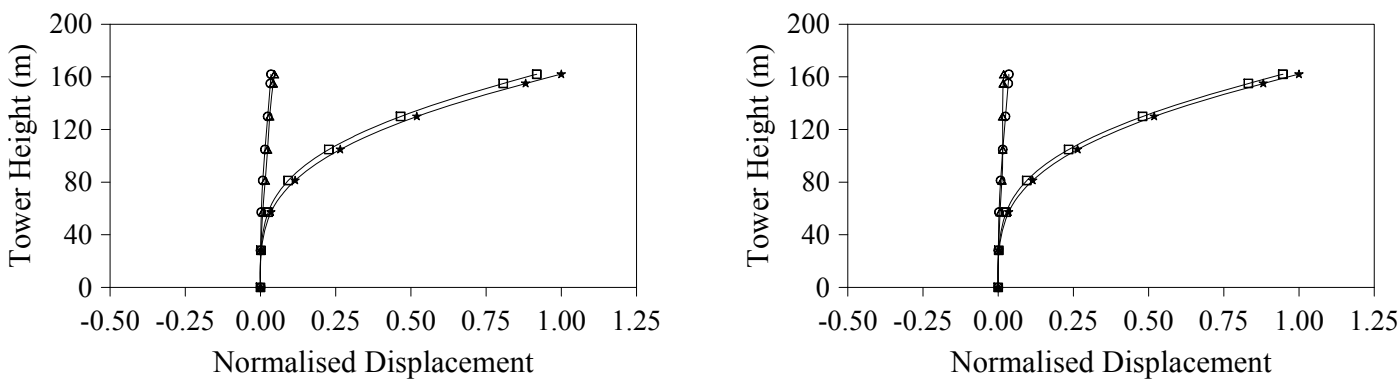

(b) European Side Tower
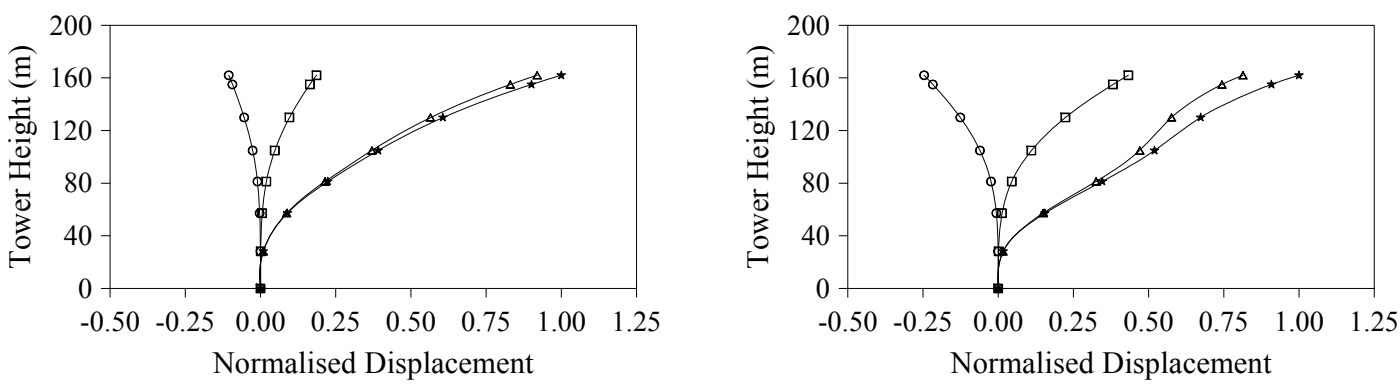

(c) Asian Side Tower

Figure 12. Normalised Stationary Displacement Variances under General Excitation for the SMFF Soil Condition Case
Figure 13. Normalised Transient Displacement Variances at $\mathrm{t}=10$ Seconds under General Excitation for the SMFF Soil Condition Case 


\section{CONCLUSIONS}

The aim of this paper is to determine the stationary and transient responses of the Bosphorus Suspension Bridge, subjected to spatially varying ground motion including the site response effect. The incoherence, wave-passage and site-response effects are examined for the spatially varying ground motion. The site-response effect is investigated in detail by locating the supports of the bridge on distinctly different soil sites. The relative contributions of the pseudo-static, dynamic and covariance components to the total response are presented for both analyses. The main findings from this study can be categorised as follows:

\section{Homogeneous and Heterogeneous Responses}

- While the stationary response values obtained for the FFFF (homogeneous) soil condition set are the lowest, the stationary response values obtained for the SMFF soil condition set are the highest. The total displacements and bending moments at the middle of the deck obtained from the general excitation case overestimates the responses by $68.39 \%$, and $98.41 \%$ and by $39.64 \%$, and $51.46 \%$ for the MMFF, and SMFF soil condition cases, respectively, when compared with the responses due to the FFFF (homogeneous) soil condition case. At the deck point where maximum shear forces take place the total shear forces obtained from the general excitation case overestimate the response by $52.94 \%$, and $67.48 \%$ for the MMFF, and SMFF soil condition cases, respectively, when compared with the response due to the FFFF soil condition case.

- While the total vertical displacements at the Asian side for the three different soil condition sets are close to each other, the displacements at the European side for the MMFF and SMFF soil condition sets are larger than those for the FFFF soil condition case.

- The response values obtained for the heterogeneous soil condition cases are larger than those of the homogeneous soil condition case. Also the greater the difference between the soil conditions, the greater the response values.

- The displacement variance values are dominated by the dynamic component at the deck and at the Asian side tower. On the other hand, while the total displacements are dominated by the dynamic component at the European side tower for the homogeneous soil condition case, the pseudo-static component dominates the total displacements for the heterogeneous soil condition cases. Furthermore, the relative contribution of the pseudo-static component to the total response generally increases as the support soil conditions weaken from firm to soft.

- While the variations obtained for the displacements at the Asian side tower for each soil condition set are similar to each other, the responses obtained at the European side tower show a different variation.

- At the top point of the European side tower where maximum total horizontal displacements take place, it can be observed that the dynamic components contributes $65.35 \%, 41.00 \%$, and $4.66 \%$; the pseudo-static component contributes $40.79 \%, 55.19 \%$ and, $91.86 \%$ and the covariance component contributes $-6.14 \%, 3.81 \%$, and $3.48 \%$ for the FFFF, MMFF, and SMFF soil condition sets, respectively. At the top point of the Asian side tower the dynamic component contributes $76.01 \%, 87.50 \%$, and $92.00 \%$; the pseudo-static component contributes $40.51 \%, 20.23 \%$, and $18.60 \%$ and the covariance component contributes $-16.52 \%,-7.73 \%$, -and $10.60 \%$ for the FFFF, MMFF, and SMFF soil condition sets, respectively.

- At the European side tower the total displacements are dominated by the dynamic component for the homogeneous FFFF soil condition case, whereas the total displacements are dominated by the pseudo-static component for the MMFF and SMFF soil condition sets. This can be attributed to the fact that the European side supports are located on weaker soil 
conditions and hence the pseudo-static components are amplified.

- The total response is dominated by the dynamic component at the Asian side tower for each soil condition case.

\section{Stationary and Transient Responses}

- The stationary response values are larger than those of the transient responses.

- For the total displacements obtained at the middle of the deck; $57.44 \%, 73.72 \%, 83.40 \%$ and $89.80 \%$ of the stationary response is achieved at the designated times of 10, 20, 30 and 40 seconds of the transient response, respectively. For the total bending moments obtained at the middle of the deck where maximum moments take place; $60.84 \%, 70.83 \%, 85.85 \%$ and $90.48 \%$ of the stationary response is achieved at the specified times of 10, 20, 30 and 40 seconds of the transient response, respectively. For the total shear forces obtained at the deck where maximum shears occur; $52.70 \%, 74.57 \%, 82.56 \%$ and $89.74 \%$ of the stationary response is achieved at the times of 10, 20, 30 and 40 seconds of the transient response, respectively.

- While the response values obtained from the transient analysis are close to those of the stationary analysis at the European side tower at the designated time of $10 \mathrm{sec}$, the response values obtained from the transient analysis are close to those of the stationary analysis at the time of $40 \mathrm{sec}$ for the Asian side tower.

- While the pseudo-static component dominates the total responses at the European side tower due to the weaker soil condition case (SM), the dynamic component dominates the total responses at the Asian side tower due to the homogeneous stiffer soil conditions (FF).

- The variation of the pseudo-static, dynamic and covariance components obtained from the transient response analyses are generally consistent with those of the stationary components. Because stationary responses are larger than those of the transient values and the pseudo-static components are equal for both responses, the contribution of the transient pseudo-static components to the total response is increased and the contribution of the transient dynamic components is decreased when compared with those of the stationary contributions.

Due to the complex nature of the problem it is difficult to make general conclusions based on a single suspension bridge model. By comparing the stationary responses with those of the transient responses obtained at various durations of the strong ground shaking used in the stationary analysis, the stationary assumption can be accepted as satisfactory for the considered ground motion duration. However, transient effects could be significant and should be taken into account in earthquakes characterized by shorter durations of strong ground shaking.

\section{REFERENCES}

[1] Abdel-Ghaffar, A.M. and Rubin, L.I., ,"Suspension Bridge Response to Multiple-Support Excitations", Journal of Engineering Mechanics, 1982, Vol. 108, pp. 419-435.

[2] Abdel-Ghaffar, A.M. and Rubin, L.I., , "Vertical Seismic Behaviour of Suspension Bridges", Earthquake Engineering and Structural Dynamics, 1983, Vol. 11, pp. 1-19.

[3] Harichandran, R.S. and Wang, W., "Response of One- and Two-Span Beams to Spatially Varying Seismic Excitation", Report to the National Science Foundation, MSU-ENGR-88-002, Michigan State University, Michigan, 1988.

[4] Bilici, Y., Bayraktar, A., Soyluk, K., Haciefendioglu, K., Ateş, S. and Adanur, S., "Stochastic Dynamic Response of Dam-Reservoir-Foundation Systems to Spatially Varying Earthquake Ground Motions”, Soil Dynamics and Earthquake Engineering, 2009, Vol. 29, pp. 444-458. 
[5] Zhang, D.Y., Liu, W., Xie, W.C. and Pandey, M.D., "Modeling of Spatially Correlated, Site-Reflected, and Nonstationary Ground Motions Compatible with Response Spectrum", Soil Dynamics and Earthquake Engineering, 2013, Vol. 55, pp. 21-32.

[6] Zembaty, Z., "Vibrations of Bridge Structure Under Kinematic Wave Excitations", Journal of Structural Engineering, 1997, Vol. 123, No. 4, pp. 479-487.

[7] Gao, Y., Wu, Y., Li, D., Liu, H. and Zhang, N., "An Improved Approximation for the Spectral Representation Method in the Simulation of Spatially Varying Ground Motions", Probabilistic Engineering Mechanics, 2012, Vol. 29, pp. 7-15.

[8] Li, B. and Chouw, N., "Experimental Investigation ff Inelastic Bridge Response Under Spatially Varying Excitations with Pounding”, Engineering Structures, 2014, Vol. 79, pp. 106-116.

[9] Der, Kiureghian, A. and Neuenhofer, A., "A Response Spectrum Method for Multiple-Support Seismic Excitations", Report No. UCB/EERC-91/08, Berkeley (CA), Earthquake Engineering Research Center, College of Engineering, University of California, 1991.

[10] Nakamura, Y., Der, Kiureghian, A. and Liu, D., "Multiple-Support Response Spectrum Analysis of the Golden Gate Bridge", Report No. UCB/EERC-93/05, Berkeley (CA), Earthquake Engineering Research Center, College of Engineering, University of California, 1993.

[11] Der, Kiureghian, A., Keshishian, P. and Hakobian, A., "Multiple Support Response Spectrum Analysis of Bridges Including the Site-Response Effect and MSRS Code", Report No. UCB/EERC-97/02, Berkeley (CA), Earthquake Engineering Research Center, College of Engineering, University of California, 1997.

[12] Rassem, M., Ghobarah, A. and Heidebrecht, A.C., , "Site Effects on the Seismic Response of a Suspension Bridge”, Engineering Structures, 1996, Vol. 18, pp. 363-370.

[13] Allam, S.M., and Datta, T.K., "Seismic Behaviour of Cable-Stayed Bridges Under Multi-Component Random Ground Motion”, Engineering Structures, 1999, Vol. 22, pp. 62-74.

[14] Allam, S.M. and Datta, T.K., "Analysis of Cable-Stayed Bridges Under Multi-Component Random Ground Motion By Response Spectrum Method”, Engineering Structures, 2000, Vol. 22, pp. 1367-1377.

[15] Soyluk, K. and Sicacik, E.A., "Soil-Structure Interaction Analysis of Cable-Stayed Bridges for Spatially Varying Ground Motion Components", Soil Dynamics and Earthquake Engineering, 2012, Vol. 35, pp. 80-90.

[16] Ateş, Ş., Soyluk, K., Dumanoglu, A.A. and Bayraktar, A., "Earthquake Response of Isolated Cable-Stayed Bridges Under Spatially Varying Ground Motions", Structural Engineering and Mechanics, 2009, Vol. 31, No. 6, pp. 639-662.

[17] Zhang, Y.H., Li, Q.S., Lin, J.H. and Williams, F.W., "Random Vibration Analysis of Long-Span Structures Subjected to Spatially Varying Ground Motions", Soil Dynamics and Earthquake Engineering, 2009, Vol. 29, No. 4, pp.620-629.

[18] Hawwari, A.R., "Suspension Bridge Response to Spatially Varying Ground Motion", Ph.D. Thesis, Michigan State University, Michigan, 1992.

[19] Harichandran, R.S., Hawwari, A. and Sweiden, B.N., "Response of Long-Span Bridges to Spatially Varying Ground Motion”, Journal of Structural Engineering, 1996, Vol. 122, No. 5, pp. 476-484.

[20] Adanur, S., Dumanoglu, A.A. and Soyluk, K., "Stochastic Analyses of Suspension Bridges: Stationary and Transient", In: Grundmann, Schueller, editors. Proceedings of the Fifth European Conference on Structural Dynamics, EURODYN 2002. Rotterdam: A.A. Balkema; 2002. 
[21] Jia, H.Y., Zhang, D.Y., Zheng, S.X., Xie, W.C. and Pandey, M.D., "Local Site Effects on a High-Pier Railway Bridge Under Tridirectional Spatial Excitations: Nonstationary Stochastic Analysis", Soil Dynamics and Earthquake Engineering, 2013, Vol. 52, pp. 55-69.

[22] Soyluk, K. and Dumanoglu, A.A., ,"Spatial Variability Effects of Ground Motions on Cable-Stayed Bridges", Soil Dynamics and Earthquake Engineering, 2004, Vol. 24, pp. 241-250.

[23] Perotti, F., "Structural Response to Nonstationary Multiple-Support Random Excitation", Earthquake Engineering and Structural Dynamics, 1990, Vol. 19, pp. 513-527.

[24] Hyun, C.H., Yun, C.B. and Lee, D.G., "Nonstationary Response Analysis of Suspension Bridges for Multiple Support Excitations”, Probabilistic Engineering Mechanics, 1992, Vol. 7, pp. 27-35.

[25] Der, Kiureghian, A., "A Coherency Model for Spatially Varying Ground Motions", Earthquake Engineering and Structural Dynamics, 1996, Vol. 25, pp. 99-111.

[26] Harichandran, R.S. and Vanmarcke, .EH., "Stochastic Variation of Earthquake Ground Motion in Space and Time", Journal of Engineering Mechanics, 1986, Vol. 112, No. 2, pp. 154-174.

[27] Clough, R.W. and Penzien, J., "Dynamics of Structures", Second Edition, Singapore: McGraw Hill, Inc., 1993.

[28] Brown, W.C. and Parsons, M.F., "Bosphorus Bridge, Part I: History of Design”, Proc. Instn Civ. Engrs, 1975, Vol. 58, pp. 505-532. 